\title{
Successful Hematopoietic Stem Cell Transplantation in AML and Hepatosplenic Candidiasis: Case Report and Review of Literature
}

\author{
Haiyan Zhang
}

Department of General Practice, The Third Affiliated Hospital of Sun Yat-Sen University, 600 Tianhe Road, Guangzhou, 510630, Guangdong Province, People's Republic of China.

\section{Corresponding Author: \\ Dr Haiyan Zhang \\ Email: 596047716@qq.com}

This is an Open Access article distributed under the terms of the Creative Commons Attribution License (creativecommons.org/ licenses/by/3.0).

Received

Accepted

July 21, 2020

Published

January 4, 2021

March 5, 2021

\begin{abstract}
Background: Hepatosplenic fungal infection in patients with acute leukemia is a rare disease. Case Report: We report a 33-year-old female with acute myeloid leukemia (AML) who developed probable hepatosplenic fungal infection after induction chemotherapy, underwent hematopoietic stem cell transplantation (HSCT) from matched sibling brother successfully. The patient received anti-fungal treatment for seven months and lesions tended to stabilize before transplantation. She was also treated with voriconazole and caspofungin throughout transplantation. Repeat abdominal MRI post-transplant revealed obvious regression of hepatic and splenic lesions. Eventually, she was discharged with full recovery of cell count. Conclusion: Complete imaging resolution of lesions is not a prerequisite for HSCT if the infection can be controlled with antifungal therapy.
\end{abstract}

Keywords: Acute Myeloid Leukemia, Antifungal Agents, Caspofungin, Hematopoietic Stem Cell Transplantation, Mycoses, Voriconazole.

\section{Introduction}

Invasive fungal infections (IFIs) are one of the most relevant causes of morbidity and mortality for patients with hematological malignancies. The epidemiology of IFIs has changed from candidemia to aspergillosis over the recent decades [1], and the rate of invasive candidiasis is estimated to be less than 1\% [2]. Chronic disseminated candidiasis (CDC) is a special clinical manifestation of invasive candidiasis; the most frequent form of CDC is hepatosplenic candidiasis (HSC). The incidence of HSC was between 2.0 and $7.4 \%$ in patients with acute leukemia [3-4].

Timely diagnosis of HSC is often difficult, because non-specific clinical presentations and limited sensitivity of diagnostic techniques including histopathology, tissue or blood culture [5-6]. At present, the optimal management of HSC has not been well established, such as the choice of therapy agents, the treatment duration. Not all guidelines are in agreement on the treatment of HSC for immunocompromised patients [7-10]. The Infectious Diseases Society of America (IDSA) suggests that treatment should be continued until lesions resolved on repeat imaging, however, which usually needs several months [11].

In addition, HSC leads to a therapeutic dilemma of whether chemotherapy or HSCT protocol should be suspended until the infection is completely eliminated, which often leads to serious consequences (i.e., relapse) [12-13]. At present, there is no related guideline on transplant indication for patients with HSC. Thus, in this study, we describe a case of HSC in a woman with AML whose liver and splenic lesions improved following seven months of three anti-fungal drugs and eventually completed subsequent HSCT from matched sibling. 


\section{Case Report}

A 33-year-old female with AML received an induction chemotherapy of cytarabine and idarubicin before admission. During agranulocytosis her main symptoms were fever and persistent low back pain. Blood culture suggested Candida tropicalis. Abdominal ultrasound revealed multiple hypoechogenic liver and splenic lesions.

In May 2017 she was transferred to our institution. Physical examination revealed conjunctival pallor, abdominal tenderness, mild hepatosplenomegaly and generalized lymphadenopathy. Repeated blood cultures for bacteria and fungus were negative. Laboratory investigation showed slightly raised liver enzymes and inflammatory indicators including procalcitonin, C-reactive protein (CRP), erythrocyte sedimentation rate (ESR), 1, 3- $\beta$-D dextran. Bone marrow examination was normal. Chest contrast enhanced computed tomography (CT) revealed multifocal lesions, mainly small nodules. An abdominal MRI demonstrated multiple low-density liver lesions partially with enhancing walls.

Based on prior Candida tropicalis fungemia and multiple low-density lesions in liver and spleen, a probable diagnosis of HSF was made. Intravenous voriconazole was initiated for the first 2 weeks, followed by combined caspofungin for the ensuing 2 weeks. On 2 June, first consolidation chemotherapy with CAG (cytarabine, aclacinomycin and granulocyte colony-stimulating factor) was initiated. Two months after dual antifungal therapy, the patient's body temperature gradually returned to normal. She was discharged to continue voriconazole $200 \mathrm{mg}$ twice daily.

About one week after discharge, the patient was readmitted for fever. Because of financial constraints, her treatment was switched to intravenous caspofungin and liposome amphotericin-B (L-AmB) for one month. Due to recurrent fever, we raised a differential diagnosis of fungus versus tuberculosis. Then except for anti-fungal drugs (caspofungin and liposome amphotericin-B), the patient received the combination of anti-tuberculosis therapy including isoniazid, rifampicin, and ethambutol. Unfortunately, after more than two months, the patient still experienced recurrent fever. Then, the patient had to receive two percutaneous fineneedle aspiration under ultrasound guidance. However, pathological examinations revealed chronic granulomatous inflammation, no fungal hyphae. Fortunately, five months after dual antifungal treatment (caspofungin and liposome amphotericin-B), repeat abdominal MRI demonstrated reduced splenic and liver lesions. She eventually underwent allo-HSCT from matched sibling brother. Intravenous voriconazole and caspofungin were given in the course of transplantation. At twenty-eight day after alloHSCT, follow-up abdominal MRI revealed reduced liver and splenic lesions and bone marrow smears indicated complete remission. She was discharged with oral voriconazole $200 \mathrm{mg}$ twice daily.

At the time of writing this paper, the patient remained stable. Her subsequent MRI revealed gradual decrease in the size of hepatic and splenic lesions.

\section{Discussion}

Invasive candidiasis infection remains the most frequent in neutropenic patients. CDC is a rare complication [14], often affecting liver, spleen, kidney and other organs. Prolonged neutropenia, glucocorticoid therapy, the existence of central venous catheters and mucositis are the most frequent risk factors of $\mathrm{CDC}$ for patients with hematological malignancies [15-16]. HSC incidence is uncertain. Some studies reported HSC rate ranged from 3\% to $7 \%$ in acute leukemia [17], and $9 \%$ in transplant recipients in Europe and North America [18]. With the use of anti-fungal prophylaxis, HSC incidence in acute leukemia may have declined below 3\% 
[19]. HSC is linked to high mortality ranging from $10 \%$ to $25 \%$ [20].

The definitive diagnosis of HSC is on the basis of tissue pathology or culture, however, with low positive rate [21]. Thus, HSC is one of the few infections where we prefer to treat rather than diagnose. In this case, antifungal treatment before biopsy may contribute to the negative pathological results. If CDC cannot be proven by microbiological examination, imaging examination is important to define probable HSC [22]. Clinically, for many patients, the presumptive diagnosis of HSF is based on imaging abnormalities. Typical imaging findings on ultrasound, CT or MRI include nodules, microabscesses ("bull-eye" lesions), hypoechogenic foci or fibrosis [23-24]. For this patient, documented Candida tropicalis and multiple hepatosplenic lesions on CT scan were sufficient to diagnose probable HSC according to the EORTC/MSG study group definition criteria [22].

There is no consensus on the drug of first choice, and the required duration of anti-fungal treatment. Most international guidelines suggest fluconazole for stable patients, and L-AmB for severely ill patients [7-9]. But Infectious Diseases Working Party of the German Society of Hematology and Oncology for hematologic patients with HSC suggested echinocandins or L-AmB as initial therapy in unstable patients, and voriconazole is considered a second-line choice [10]. In this case, for kinds of reasons, the patient was rendered subsequently three anti-fungal agents including voriconazole, echinocandins and L-AmB. Clinically, specific therapeutic drugs depend on a variety of factors including severity of infection, host immune state, previous anti-fungal regimen, patient's tolerance and economic capability. Hence, it is difficult to answer the question: what is the best treatment for patients with HSC. Another, we should be aware that fever can persist for several weeks, even several months; and it is not usually considered as a failure of treatment during the evolution of HSC [25-26].
The Infectious Diseases Society of America advises that treatment should be continued until radiologic lesions are calcified or resolved, which usually needs several months. However, others consider anti-fungal therapy should be continued throughout chemotherapy course or HSCT [27]. In this case, the patient received anti-fungal therapy throughout transplantation.

But no consensus on the transplant time for patients with HSC was reached. Historically, uncontrolled fungal infection was considered a relative contraindication to HSCT. However, improvement in transplant method and posttransplant supportive technology have made HSCT possible for patients with disseminated fungal infection [28]. In the case, reduced intensity transplant (RIT) and HSC in a stable state before HSCT prevented fungal progression after transplant. CDC was not an absolute contraindication to stem cell transplantation [3,29]. One study reported 22 of the sixty-one patients with HSC underwent allogeneic transplantation successfully after HSC was controlled [30].

\section{Conclusion}

HSC remains a challenging issue for patients with hematological malignancies. Timely diagnosis of HSC is challenging due to the low yield rate of positive microbiological and histological findings. In case of patients with persistent and unexplained fever and hepatic and splenic lesions, HSC should be considered when lesions are not characterized by metastasis. The encouraging outcome confirms HSC is not an absolute contraindication to chemotherapy schedule and HSCT, if the lesions are stable.

\section{References}

1. Pagano L, Caira M, Rossi G, Tumbarello M, Fanci R, Garzia MG, et al. Hema e-Chart Group I (2012). A prospective survey of febrile events in hematological malignancies. Ann Hematol. 2012;91(5):767-774.

2. Gamaletsou MN, Walsh TJ, Zaoutis T, Pagoni M, Kotsopoulou M, Voulgarelis M, et al. A prospective, 
cohort, multicentre study of candidaemia in hospitalized adult patients with haematological malignancies. Clin Microbiol Infect. 2014;20:O50-57.

3. Chen CY, Chen YC, Tang JL, Yao M, Huang SY, Tsai $\mathrm{W}$, et al. Hepatosplenic fungal infection in patients with acute leukemia in Taiwan: incidence, treatment, and prognosis. Ann Hematol. 2003;82:93-97.

4. Karthaus M, Hebart H, Einsele H, Schaefer H, ScheelWalter $\mathrm{H}$, Buchheidt $\mathrm{D}$, et al. Long-term survival in patients with acute leukemia and chronic disseminated candidiasis despite minimal antileukemic therapy. Haematologica. 2006;91:1422-1423.

5. Cuenca-Estrella M, Verweij PE, Arendrup MC, ArikanAkdagli S, Bille J, Donnelly JP, et al. ESCMID guideline for the diagnosis and management of Candida diseases 2012: Diagnostic procedures. Clin Microbiol Infect. 2012;18:9-18.

6. Leroy O, Gangneux JP, Montravers P, Mira JP, Gouin F, Sollet JP, et al. Epidemiology, management, and risk factors for death of invasive Candida infections in critical care: A multicenter, prospective, observational study in France (2005-2006). Crit Care Med. 2009;37:16121618.

7. Pappas PG, Kauffman CA, Andes D, Benjamin DK Jr, Calandra TF, Edwards JE Jr, et al. Clinical practice guidelines for the management of candidiasis: 2009 update by the Infectious Diseases Society of America. Clin Infect Dis. 2009;48(5):503-535.

8. Chakraborty KK, Naik SR. In situ liposomal preparation containing amphotericin B: related toxicity and tissue disposition studies. Pharm Dev Technol. 2000;5(4):543553.

9. Cornely OA, Bassetti M, Calandra T, Garbino J, Kullberg BJ, Lortholary O. Group EFIS (2012) ESCMID guideline for the diagnosis and management of Candida diseases 2012: non-neutropenic adult patients. Clin Microbiol Infect. 2012;18(Suppl 7):19-37.

10. Mousset S, Buchheidt D, Heinz W, Ruhnke M, Cornely $\mathrm{OA}$, Egerer $\mathrm{G}$, et al. Treatment of invasive fungal infections in cancer patients-updated recommendations of the Infectious Diseases Working Party (AGIHO) of the German Society of Hematology and Oncology (DGHO). Ann Hematol. 2014;93(1):13-32.

11. Pappas PG, Kauffman CA, Andes DR, Clancy CJ, Marr KA, Ostrosky-Zeichner L, et al. Clinical practice guideline for the management of Candidiasis: 2016 update bythe Infectious Diseases Society of America. Clin Infect Dis. 2016;62:e1-50.

12. Lehrnbecher T, Frank C, Engels K, Kriener S, Groll AH, Schwabe D. Trends in the postmortem epidemiology of invasive fungal infections at a university hospital. J Infect. 2010;61(3):259-265.

13. Masood A, Sallah S. Chronic disseminated candidiasis in patients with acute leukemia: emphasis on diagnostic definition and treatment. Leuk Res. 2005;29(5):493-501.

14. Blade J, Lopez-Guillermo A, Rozman C, Grañena A, Bruguera M, Bordas J, et al. Chronic systemic candidiasis in acute leukemia. Ann Hematol. 1992;64:240-244.

15. Bergamasco MD, Garnica M, Colombo AL, Nucci M. Epidemiology of candidemia in patients with hematologic malignancies and solid tumours in Brazil. Mycoses. 2013;56:256-263.

16. Puig-Asensio M, Ruiz-Camps I, Fernández-Ruiz M, Aguado JM, Muñoz P, Valerio M, et al. Epidemiology and outcome of candidaemia in patients with oncological and haematological malignancies: RESULTS from a population-based surveillance in Spain. Clin Microbiol Infect. 2015;21:e1-e10.

17. Cornely OA, Ullmann AJ, Karthaus M. Evidence-based assessment of primary antifungal prophylaxis in patients with hematologic malignancies. Blood 2003;101:33653372.

18. Sallah S, Semelka RC, Wehbie R, Sallah W, Nguyen NP, Vos P. Hepatosplenic candidiasis in patients with acute leukemia. Br J Hematol. 1999;106:697-701.

19. Rammaert B, Desjardins A, Lortholary O. New insights into hepatosplenic candidosis, a manifestation of chronic disseminated candidosis. Mycoses. 2012;55:e74-e84.

20. Groll AH, Castagnola E, Cesaro S, Dalle JH, Engelhard $\mathrm{D}$, Hope W, et al. Fourth European Conference on Infections in Leukaemia (ECIL-4): guidelines for diagnosis, prevention, and treatment of invasive fungal diseases in paediatric patients with cancer or allogeneic haemopoietic stem cell transplantation. Lancet Oncol. 2014; 15:e327-e340.

21. Ullmann AJ, Akova M, Herbrecht R, Viscoli C, Arendrup MC, Arikan-Akdagli S, et al. ESC-MID guideline for the diagnosis and management of Candida diseases 2012: Adults with haematological malignancies and after haematopoietic stem cell transplantation (HCT). Clin Microbiol Infect. 2012;18:53-67.

22. de Pauw B, Walsh TJ, Donnelly JP, Stevens DA, Edwards JE, Calandra T, et al. Revised definitions of invasive fungal disease from the European Organization for Research and Treatment of Cancer/Invasive Fungal Infections Cooperative Group and the National Institute of Allergy and Infectious Diseases Mycoses Study Group (EORTC/MSG) Consensus Group. Clin Infect Dis. 2008;46:1813-1821.

23. Marchetti O, Lamoth F, Mikulska M, Viscoli C, Verweij P, Bretagne S. ECIL recommendations for the use of biological markers for the diagnosis of invasive fungal diseases in leukemic patients andhematopoietic SCT recipients. Bone Marrow Transplant. 2012;47:846-854.

24. Senn L, Robinson JO, Schmidt S, Knaup, M Asahi $\mathrm{N}$, Satomura S, et al. 1,3-Beta-D-glucanantigenemia 
for early diagnosis of invasive fungal infections inneutropenic patients with acute leukemia. Clin Infect Dis. 2008; $46: 878-885$.

25. Anaissie E, Bodey GP, Kantarjian H, David C, Barnett K, Bow E, et al. Fluconazole therapy for chronic disseminated candidiasis in patients with leukemia andprior amphotericin B therapy. Am J Med. 1991;91:142-150.

26. Kauffman CA, Bradley SF, Ross SC, Weber DR. Hepatosplenic candidiasis: Successful treatment with fluconazole. Am J Med. 1991;91(2):137-141.

27. Pappas PG, Kauffman CA, Andes DR, Clancy CJ, Marr KA, Ostrosky-Zeichner L, et al. Clinical practice guideline for the management of candidiasis: 2016 update by the Infectious Diseases Society of America. Clin Infect Dis. 2016;62:e1-e50.
28. Aki ZS, Sucak GT, Yeğin ZA, Guzel O, Erbas G, Senol E. Hematopoietic stem cell transplantation in patients with active fungal infection: not acontraindication for transplantation. Transplant Proc. 2008;40:1579-1585.

29. Bjerke JW, Meyers JD, Bowden RA. Hepatosplenic candidiasis-a contraindication to marrow transplantation? Blood. 1994;84:2811-2814.

30. Chien-Yuan Chen, Aristine Cheng, Feng-Ming Tien, Po-Chu Lee, Hwei-Fang Tien, Wang-Huei Sheng, et al. Chronic disseminated candidiasis manifesting as hepatosplenic abscesses among patients with hematological Malignancies. BMC Infectious Diseases. 2019;19:635. 